\title{
PROPUESTA DE UN REFERENTE CONCEPTUAL PARA POSICIONARSE FRENTE A LOS RETOS DE LA EDUCACIÓN SECUNDARIA Y ALGUNOS LINEAMIENTOS PARA ORIENTAR SU REFORMULACIÓN
}

\author{
Ileana Contreras Montes de Oca \\ Investigadora y docente de la Universidad de Costa Rica.
}

Recibido 14-XI-2005

Aceptado 25-IV-2006

\begin{abstract}
Resumen: El artículo es producto de un análisis bibliográfico acerca del tema, el cual sustenta la propuesta y los lineamientos derivados de éste. Recoge los diversos retos, dilemas y desafíos que se han venido planteando a la educación secundaria, tanto en el contexto mundial, como en el Latinoamericano $y$ del Caribe, en el marco de las transformaciones del contexto socioeconómico, político y cultural en que se desarrolla. Plantea la necesidad de abordar la lectura del amplio mapa de expectativas existentes respecto de dicho nivel educativo, desde un referente conceptual integrador de las distintas miradas y enfoques con que se han abordado sus problemas. Esboza algunos conceptos que deben incluirse en este referente. Finalmente, a manera de conclusión, sobre la base de los conceptos propuestos, establece algunos lineamientos para orientar los esfuerzos dirigidos a atender las diversas demandas y retos a las que actualmente se enfrenta la educación secundaria costarricense.
\end{abstract}

Palabras clave: Educación secundaria, demandas, retos, cambios.

\section{Introducción}

En la mayoría de los países del mundo, incluyendo los de la región Latinoamericana, la educación secundaria es el centro de análisis y de propuestas de reforma, así como de búsqueda de alternativas orientadas a responder a las muy diversas expectativas que se formulan en torno a ella.

La historia de la educación da cuenta del hecho de que cada vez que se visualiza o se adquiere conciencia de la necesidad de mejorar su papel en el entramado social, se realizan importantes esfuerzos de análisis para generar alternativas de cambio o de reforma. Sin embargo, tradicionalmente tales esfuerzos se han dirigido a la educación primaria o a la que denominamos básica. Nunca, como en el presente, se le ha dado a la educación secundaria el lugar y la atención que hoy se evidencia. Indudablemente ello responde a las características particulares de las transformaciones gestadas en su entorno.

Es así como en la actualidad, se presencia una importante preocupación por la educación secundaria, así como planteamientos en torno a la necesidad de que esta logre: 


\begin{abstract}
This article is a result of a bibliographical analysis as the basis for the conceptual frame and guidelines proposed. It summarizes the challenges and dilemmas actually faced by the secondary school level in the world, and Latin American and the Caribbean as well, in the context of the socio, economical, political and cultural changes in which it takes place. The study states the need to approach the range of expectations existing around this educational level with an integral conceptual frame of reference including the different perspectives and approaches with which its problems have been faced. Finally, the study offers some orientations to face the most important challenges and demands in Costa Rican secondary education.
\end{abstract}

Key words: Secondary school, challenges, demands, changes.
1. La universalización o ampliación del acceso, así como mejorar la retención y el rendimiento

2. Promover el desarrollo de competencias y capacitación para el trabajo

3. Mejores aprendizajes dentro de instituciones y sistemas más efectivos y eficientes

4. Desarrollar la capacidad de aprender a aprender, de búsqueda y análisis crítico de la información que cada vez es más abundante y de fácil acceso

5. La flexibilización de los currículos en estrecha vinculación con la diversidad de la población que accede a ella

6. Una adecuada articulación con el entorno y con los otros componentes del Sistema

7. La incorporación de la tecnología para mejorar su calidad

8. Una reestructuración organizativa que le permita responder a las demandas que se le plantean

9. Condiciones de trabajo, recursos y materiales apropiados, lo cual requiere de financiamiento adecuado

10. El desarrollo de innovaciones y de investigaciones que estudien las diversas alternativas para incorporar los cambios que se esperan, y para el análisis de aquellos aspectos que funcionan o han tenido éxito en condiciones particulares.

Por lo tanto, en este artículo se recoge el resultado de un análisis bibliográfico orientado a responder a las siguientes interrogantes:

¿A qué responde ese interés? ¿Cómo se ha transformado el entorno de la educación secundaria y qué implicaciones tienen dichas transformaciones en ésta?

¿Cuáles perspectivas pueden orientar la lectura de los retos que se plantean? ¿Qué aspectos deben tenerse en cuenta al identificar los retos y alternativas a nuestro alcance? ¿Desde dónde establecer las prioridades? ¿Cuáles son los retos que debe asumir la educación secundaria costarricense 
prioritariamente? ¿Qué condiciones deben satisfacer las estrategias que se propongan para asumirlos?

Para ello, se incluye en primer término, una reseña de las grandes transformaciones a las que asistimos en el nivel mundial y de las formas en que estas impactan de manera particular el ámbito socioeconómico, político y cultural de los países y sus organizaciones, así como a los diversos sectores dentro de ellos, tanto en el nivel institucional como en el personal, en los distintos contextos histórico geográficos.

Seguidamente, se hace referencia a los principales dilemas y retos que estas transformaciones han venido planteando a los sistemas educativos en general y a la educación secundaria en particular, según se reporta en la bibliografía reciente.

Luego, se destaca la necesidad e importancia de establecer claramente las perspectivas desde donde se hará la lectura de los dilemas y retos que se plantean a la educación secundaria, y el análisis de sus implicaciones en nuestro país. Se plantea que dichas perspectivas deben constituir un referente conceptual integrador de las distintas miradas y enfoques con que se han abordado sus problemas en nuestro medio, y se esbozan algunos conceptos que se propone incluir en dicho referente.

Finalmente, con base en las perspectivas propuestas para su lectura, se establecen algunos lineamientos para orientar los esfuerzos dirigidos a atender las diversas demandas y retos que actualmente enfrenta la educación secundaria costarricense.

\section{II. ¿Cómo se transforma nuestro mundo y de qué manera se viven los cambios en los contextos histórico geográficos?}

Los distintos países y regiones del mundo se enfrentan hoy a desafíos que no son sólo económicos y tecnológicos, sino también culturales en el sentido amplio.
Entre los más importantes se encuentra la tarea de educar a una nueva generación para vivir en un mundo atravesado por un cambio tan rápido que puede calificarse de "revolucionario".

La expansión de la base de conocimiento ha generado como consecuencia, una variación radical de nuestra forma de vida. Los cambios tecnológicos no sólo han alterado las formas de producción y distribución de sus frutos, sino además, están alterando la propia textura de la vida humana. En el entorno social se aprecian diversos reacomodos políticos, que han variado las relaciones entre los países, convirtiendo al mundo en un mercado de proporciones descomunales. Se han generado nuevas necesidades.

La conservación de muchos grupos humanos y de muchas especies animales y vegetales $-\mathrm{y}$ en general del medio ambiente- se ve amenazada. Las nuevas condiciones morales y materiales demandan atender problemas cuantitativa y cualitativamente muy distintos a los de la época en que se definió el modelo educativo en general, y el de la educación secundaria en particular (Hargreaves, 96; Puiggrós, 94; Krauskopf, 2001; Contreras, 2002).

Entre los cambios que caracterizan el entorno actual con mayor incidencia en el campo educativo, pueden citarse los siguientes:

- Rompimiento de las barreras de espacio y tiempo. Se posibilita la comunicación y la toma de decisiones en diversos lugares y en forma simultánea.

- Incremento acelerado del conocimiento y la información, lo cual demanda el desarrollo de habilidades para seleccionar y analizar lo que es relevante para cada objetivo; y torna irrelevante e inalcanzable, la pretensión de que un individuo pueda albergar o apropiarse de todo lo disponible.

- La aparición de economías basadas en el conocimiento. 
- Modificación de la estructura ocupacional debido a la incorporación de técnicas y procesos laborales más flexibles y tecnológicamente más desarrollados, que requieren trabajadores con nuevas cualificaciones. Inestabilidad del empleo y requerimientos cambiantes para los puestos de trabajo disponibles, entre ellos el manejo de al menos una segunda lengua y de programas de computación.

- $\quad$ Predominio de la imagen visual por sobre las otras formas de representación, lo cual ha generado una cultura visual instantánea, suplantando la reflexión estudiada, el debate público y el discurso moral.

- La transformación del Estado -o reducción de su tamaño- así como de su papel de "benefactor" pone bajo escrutinio el gasto público, incluyendo el asignado a la educación, propiciando la iniciativa privada en ámbitos que anteriormente eran de carácter público.

- La desconfianza ciudadana en sus órganos de representación y en muchas de las instituciones tradicionales y la llamada "crisis de gobernabilidad".

- Demanda por organizaciones menos burocráticas y compartamentalizadas, sin largas cadenas de mando que retrasan las decisiones y limitan la posibilidad de aprovechar las oportunidades. Se requieren organizaciones caracterizadas por funciones y unidades flexibles e inter-comunicadas.

- Desarrollo de la Telemática.

- Cambios acelerados en el entorno físico.

El mundo en el que actualmente se insertan los sistemas educativos se ha transformado en un mundo globalizado caracterizado por cuatro poderes: el económico, el tecnológico, el informático y el cultural. Algunos lo describen como una época histórica de desarrollo estructuralmente desequilibrado y socialmente excluyente, en tanto que otros lo califican como un dualismo que nos muestra dos mundos separados dentro de las fronteras de un mismo país (Roldán, 2001).

En su libro, The Work of Nations, Robert Reich (1992) afirma:

Estamos viviendo una transformación que reestructurará la política y la economía del siglo próximo. No habrá productos ni tecnologías nacionales ni corporaciones nacionales ni industrias nacionales. Ya no habrá economías nacionales, al menos tal y como entendemos ese concepto. Sólo las personas que constituyen la nación permanecerán enraizadas dentro de las fronteras nacionales. Los principales activos de cada nación serán las destrezas e intuiciones de sus ciudadanos. La principal tarea política de cada nación será contrarrestar las fuerzas centrífugas de la economía global que rompe los lazos que unen a los ciudadanos -proporcionando cada vez más riqueza a los más preparados y perspicaces y destinando a los menos adiestrados a un nivel de vida decadente-. A medida que las fronteras vayan teniendo cada vez menos sentido en términos económicos, los ciudadanos mejor situados para prosperar en el mercado mundial tendrán la tentación de desembarazarse de los vínculos de lealtad nacional y, en consecuencia, de desentenderse de sus compañeros menos favorecidos (p. 3).

En las economías industrializadas, el patrón tradicional de los logros académicos consistía en centrarse en la educación de las élites, de forma que el nivel medio de rendimiento y el rendimiento de aquellos que obtenían altos niveles de logros educativos era lo que impulsaba el crecimiento económico. A medida que aumentan los requisitos técnicos en el caso de los trabajos que ofrecen un nivel de vida decente, el diferencial en el rendimiento educativo se vuelve tan importante, si no más, que el nivel medio de rendimiento, o el rendimiento de las personas que obtienen los más altos logros educativos. Entre más dependientes de la distribución de las destrezas y del conocimiento en la economía, las políticas buscan encaminarse a la reducción de las diferencias en el acceso al trabajo académico exigente y el rendimiento que resulta de tal trabajo (Elmore, 2003).

Por otra parte, dichas transformaciones socioeconómicas y políticas han tenido un fuerte impacto en las modificaciones y 
vinculaciones que las personas establecen con su entorno y en la impronta que caracteriza el desarrollo juvenil. Ha disminuido el dominio directo de la familia y la educación como agencias socializadoras, sobre la vida adolescente (Krauskopf, 2001). La rapidez de los cambios ha conducido a códigos diferentes para interpretar la realidad entre las generaciones, lo cual permite referirse a una transición cultural hacia lo que Mead (1971) denominaba cultura "prefigurativa" en la que, a diferencia de las épocas anteriores, el modelo para los jóvenes depende culturalmente en menor grado de los adultos; en donde los pares aportan al compartir la exploración del mundo desde nuevas dimensiones. Los padres sienten que su propio pasado es inapropiado para orientar el futuro de sus hijos. En comparación con los ambientes de trabajo de sus padres, seguros, estables y, a veces monótonos, las nuevas generaciones trabajan en contextos organizacionales muy diferentes, de carácter más reducido, menos seguro y menos estable.

En este contexto, la tarea de las nuevas generaciones es aprender a vivir no sólo en el amplio mundo de una tecnología cambiante y un flujo continuo de información, sino ser capaces, al mismo tiempo, de mantener y refrescar también sus identidades locales. Por lo tanto, ni la escuela ni la educación pueden concebirse ya como meros vehículos de transmisión de habilidades básicas que se requieren para ganarse la vida o para mantener la competitividad económica de los respectivos países. Para que esta dimensión económico-tecnológica de nuestro mundo sea viable tiene que encuadrarse en un contexto cultural humano que la sostenga. La tarea central es, entonces, crear un mundo que dé significado a nuestras vidas, a nuestros actos, a nuestras relaciones. Del mismo modo que aprendemos a trabajar juntos, tenemos que aprender a aprender de los otros, a compartir esfuerzos para comprender el mundo personal, social y natural.

\section{I. ¿Cuáles son los principales retos y dilemas que se viene planteando a la educación secundaria?}

Las condiciones que dan sentido a la oferta educativa y los supuestos que la sustentan han sufrido importantes transformaciones en el transcurso del desarrollo histórico de su institucionalización. En el contexto mundial, la cobertura de la educación primaria se ha tornado obligatoria, y la educación secundaria ha dejado de ser una instancia de minorías (Krauskopf, 2001).

Lo anterior, por cuanto las transformaciones y cambios en el contexto de la educación secundaria, descritos en el apartado anterior, necesariamente repercuten en los sistemas educativos en general. Sin embargo, su impacto resulta más decisivo para la educación secundaria general, debido al papel que se espera que ésta desempeñe tanto en el desarrollo de destrezas y competencias requeridas del personal que demanda el aparato de producción, como en la formación de los ciudadanos que han de constituir las sociedades actuales (Leclercq, 2001).

Por otra parte, la educación secundaria se ha dirigido tradicionalmente a atender las necesidades educativas de la población adolescente-juvenil, y hoy día esta es motivo de mayor interés y preocupación, debido no sólo al hecho de que representa un importante sector, sino especialmente porque existe una mayor conciencia de las capacidades, las potencialidades, las necesidades y las dificultades que tiene y que deberá enfrentar en el futuro próximo (Donas, 2001).

En el desarrollo histórico de la oferta educativa es posible identificar los siguientes momentos destacados por Obiols y Obiols (1995): Su importancia emerge en el siglo XVIII, razón por la cual se le conoce como el siglo de las luces; en éste se plantea la educabilidad humana. En el siglo XIX el sistema de enseñanza adquiere su carácter 
público y estatal, y se orienta a la formación del ciudadano con una función económico social. En la segunda mitad del siglo $\mathrm{XX}$, en los países de mayores recursos, la educación secundaria pasa a generalizarse con el objetivo de ofrecer a los individuos un marco cultural para comprender y para desenvolverse en el mundo. Dicha base, hasta mediados de siglo, la ofreció la escuela primaria; sin embargo, hoy es a la escuela secundaria -o a la "educación general básica"- a la que le corresponde brindarla.

En la región Latinoamericana la educación secundaria constituía, hasta hace poco, el nivel de educación "olvidado". Sin embargo, actualmente existe un fuerte movimiento dirigido a la educación secundaria (Wolf y de Moura, 2000).

En el plano nacional es posible apreciar una preocupación cada vez mayor por la educación secundaria. El Undécimo Informe sobre el Estado de la Nación en Desarrollo Humano Sostenible, entregado recientemente, incluye un informe especial orientado a señalar la urgente necesidad de universalizar la educación secundaria (CONARE, 2005).

Como producto de este interés creciente por la educación secundaria, se han venido desarrollando, tanto en el ámbito internacional como en el Latinoamericano, esfuerzos orientados a determinar y analizar los retos y desafíos que enfrenta este nivel educativo en el mundo actual. A continuación, se presenta una síntesis de los planteamientos de dos documentos que directamente abordan esta temática, uno en el nivel mundial y el otro en el Latinoamericano y del Caribe, los cuales analizan de manera comprensiva las diversas posiciones que al respecto se han venido esbozando.

El primero de ellos corresponde a la Reunión de Expertos Internacionales sobre la Educación Secundaria en el Siglo XXI de la UNESCO, celebrada en Beijing, República Popular de China, en mayo 2001, en la cual se llegó al siguiente consenso:
- La necesidad de dar prioridad a la Educación Secundaria

- La necesidad de redefinir los objetivos y las funciones de la Educación Secundaria para el siglo XXI.

El eje de la reunión giró alrededor de los grandes dilemas ante los que ésta se encuentra o enfrentará en el futuro, para poder alcanzar los nuevos objetivos y funciones que se le asignan. Tales dilemas son:

1. La educación secundaria masiva o selectiva

2. Una orientación general o una educación especializada -formación profesional-

3. Búsqueda de resultados en el plano cognitivo o en el plano de las actitudes

Al respecto, los principales acuerdos establecidos son los siguientes:

1. Respecto del dilema de la masificación o la selectividad: Un compromiso con la masificación y universalización de la educación secundaria apoyado con iniciativas destinadas a la supresión de las barreras y obstáculos, especialmente para las jóvenes, y con base en la utilización de los medios y oportunidades existentes. Ello requiere ofrecer una atención preferente y una oferta adecuada a aquellos alumnos excluidos de ella y a los que tengan dificultades en la transición de un ciclo a otro. Para lograrlo, se requiere la colaboración entre los gobiernos y otros sectores -privados, ONG-; sin embargo, es responsabilidad de los gobiernos garantizar el mismo nivel de calidad en toda la educación secundaria. También, es necesario reestructurar los programas de manera que ofrezcan mayor flexibilidad y posibilidad de elección a los estudiantes. Asimismo, la educación secundaria debe orientarse hacia una 
mayor calidad y diversificación de su oferta, una mayor flexibilidad en la organización de la enseñanza, y una mayor adecuación a las necesidades y circunstancias de los estudiantes.

2. En relación con el dilema entre generalización o especialización: Se plantea la necesidad de centrarse en las relaciones entre las materias académicas y las profesionales y su interdependencia en la educación general de los jóvenes de ambos sexos. Puesto que la finalidad de la educación secundaria es la educación para la vida, ésta debe reflejar la realidad en que se inserta en el siglo XXI. Ello torna necesario un ir y venir continuo entre el aprendizaje y el mundo del trabajo. La importancia que se confiere a las materias profesionales está determinado por la índole que éstas tengan, la forma en que se organizan y enseñan, así como por su reconocimiento en el currículo. Tanto la educación secundaria como la formación profesional debe orientarse a atender no sólo las necesidades sociales en materia de recursos humanos, sino además las necesidades de desarrollo y aspiraciones individuales. En la mayoría de los países existe una firme tendencia a incluir materias profesionales en la educación secundaria dirigida a todos los estudiantes. En algunos otros, se está probando ofrecer este tipo de formación conjunta a distintos grupos, como migrantes, poblaciones rurales aisladas y estudiantes de secundarias corrientes. Puesto que la incorporación de este tipo de formación puede resultar cara, y por lo tanto, provocar logros de baja calidad y hasta un descenso de su consideración, se han probado estrategias innovadoras y asequibles para evitarlo, como: contratar la enseñanza de estas asignaturas con escuelas o centros especializados próximos a las instituciones; generar nuevos métodos didácticos para la enseñanza de estas materias que requieran menos equipamiento, materiales e instructores con experiencia. Finalmente, y dado que la educación permanente se perfila con claridad para adaptarse a esta realidad, la educación secundaria está desarrollando unos programas de estructuras flexibles y diversificadas, y con más estrechas relaciones con el mundo del trabajo.

3. $\quad$ Con respecto al dilema de procurar resultados en el plano cognitivo o en el plano de las actitudes: Se coincidió en reconocer que la educación secundaria tradicional no atiende adecuadamente las necesidades de los estudiantes para lograr la realización de todo su potencial, especialmente en un contexto de rápidos cambios económicos, culturales y sociales, así como de discriminación entre los sexos. Ante la pérdida de influencia de otras entidades socializantes, las escuelas deben asumir cada vez más la responsabilidad de propiciar la preparación necesaria para la vida activa. Para ello, resulta esencial la nueva función de los docentes -como mediadores-, su condición social, su integridad, y su compromiso. Por lo tanto, la calidad de la formación del profesorado, inicial y permanente, adquiere gran importancia. Así mismo, la función de los directores de estas instituciones debe ser coherente con esta nueva realidad. Además, se genera la necesidad de desarrollar y adaptar los métodos didácticos, los recursos, y los servicios escolares para la formación de actitudes y para la preparación para la vida activa. De ello se desprende el requerimiento de que los responsables de las políticas educativas estén conscientes de las repercusiones de sus decisiones y las 
respalden en factores reales, como la relación entre el éxito académico y el bienestar social y de la comunidad.

Además, para la incorporación exitosa de este tipo de educación se requiere adoptar un enfoque multi-sectorial que involucre los ministerios, las comunidades locales, organizaciones no gubernamentales, entre otros.

Otros temas identificados en la Reunión como claves para que la educación secundaria pueda cumplir con estos nuevos objetivos y funciones, son los siguientes:

- La educación integrada y compensatoria

- La eliminación de las desigualdades de género

- La educación holística y participativa

- $\quad$ El fortalecimiento de la equidad

- El uso efectivo de las tecnologías de la información y la comunicación

A partir del reconocimiento de que los alumnos son el centro de toda reforma educativa y de que existen tres elementos interrelacionados que favorecen su aprendizaje: las personas -profesionales de la educación, padres o miembros de la comunidad-; las políticas educacionales, que ofrecen un marco para delimitar lo que los alumnos deben aprender; y la infraestructura, las naciones miembros pidieron la colaboración de la UNESCO en las siguientes tareas:

1. En relación con los alumnos: Replantear la Educación Secundaria para que ésta responda a las necesidades diversas de los educandos y para que se les ofrezcan los conocimientos necesarios, así como la preparación adecuada para la vida activa, incluyendo el aprendizaje profesional.

2. Con respecto a los docentes, el currículo y la escuela: Dar prioridad a la formación del profesorado -inicial y en servicio-; garantizar que los currículos expresen con claridad y precisión las finalidades y sus objetivos reflejen las necesidades locales, sin olvidar las socioeconómicas; y finalmente, asegurar que las escuelas se orienten a convertirse en comunidades de aprendizaje, por lo que su organización debe adaptarse a la función: requieren nuevas formas de trabajo, flexibles y simplificadoras; equipos educativos abiertos a otras personas, además de los docentes; y formas nuevas, democráticas y descentralizadas de dirigir las escuelas.

3. En cuanto a la política e infraestructura: Garantizar que quienes definen las políticas educativas presten atención suficiente a la educación secundaria; posibilitar el acceso a este nivel educativo para todos los alumnos; proporcionarle, por medio de asignaciones presupuestarias adecuadas, los recursos que requiere.

El otro documento utilizado como referente para derivar la información disponible y reciente, en relación con los planteamientos relativos a la educación secundaria en la región Latinoamericana y del Caribe, corresponde a la serie de informes técnicos del Departamento de Desarrollo Sostenible del Banco Interamericano de Desarrollo. Se trata del documento titulado Educación secundaria en América Latina y el Caribe; los retos del crecimiento y la reforma (Wolff y de Moura, 2000).

Dicho documento se inicia destacando el papel fundamental que adquiere la educación secundaria, en un entorno caracterizado por un creciente contenido intelectual de la producción, que exige una fuerza laboral con muy sólidas habilidades en matemáticas, lenguaje y comunicación, así como con mayor flexibilidad, creatividad y capacidad para el trabajo conjunto. Con base en dicho reconocimiento, establece seis áreas críticas para el mejoramiento 
de la calidad de la educación en ese nivel y propone una agenda tentativa para su crecimiento y reforma. Para ello, incluye una descripción de la situación actual de la educación secundaria en la Región, producto de la síntesis que realizan los autores acerca de los temas relacionados con la calidad de educación secundaria, de los problemas que enfrenta, y de investigaciones y mejores prácticas que actualmente se adelantan tanto en la Región como en otros lugares.

Los siguientes son los rasgos principales de su caracterización de la situación actual:

Las transformaciones socioeconómicas en las que se insertan los sistemas educativos han generado en los países desarrollados una educación secundaria prácticamente universal, la revisión de los currículos, y estándares de aprendizaje más elevados. En América Latina y el Caribe, por el contrario, el porcentaje de la matrícula es mucho menor que el de los principales competidores de la Región. El aprendizaje en la educación secundaria es insuficiente, según criterios internacionales.

Los currículos que se implementan son obsoletos y no corresponden a las necesidades del mercado laboral. Los profesores poseen un conocimiento insuficiente de los temas; utilizan pedagogías inadecuadas y, a menudo, están poco motivados. Los materiales de aprendizaje resultan escasos e inadecuados; las escuelas rara vez poseen un sentido de misión e identidad, y sus directores obtienen, por lo general, poca autoridad y reconocimiento.

Estos problemas se agravan ante el creciente número de estudiantes que ingresan a la educación secundaria, provenientes de contextos sociales diferentes y con necesidades diversas, en comparación con el tipo de estudiantes que anteriormente accedía a ella, en un sistema relativamente más elitista. Además, los pobres y las minorías, sobre todo en las áreas rurales, están insuficientemente representados; una gran cantidad de jóvenes adultos asisten todavía a la escuela secundaria, como resultado de la repetición de niveles anteriores y en la escuela secundaria.

Por lo tanto, establece seis áreas críticas para el mejoramiento de la calidad de la educación secundaria en la Región:

1. Mayor concentración en el aprendizaje y en la medida de rendimiento: Una mejora cualitativa significa mayor aprendizaje en habilidades de orden superior en matemáticas, comunicaciones y lenguaje. A pesar de la introducción de pruebas nacionales en casi todos los países latinoamericanos, el reporte de resultados no garantiza mayor aprendizaje. Por lo tanto, es necesario articular criterios claros, diseñar formas de evaluación apropiadas, y estrategias coherentes para el uso de los resultados.

2. Enseñanza más efectiva, mejores profesores y reforma de la capacitación docente: Una mayor dedicación de tiempo al aprendizaje y menor a los procesos repetitivos y a las rutinas administrativas. Profesores con mejor conocimiento de las asignaturas a su cargo y con la capacidad de modificar sus enfoques pedagógicos para alcanzar diferentes objetivos de aprendizaje con distintos grupos de estudiantes. Es fundamental, entonces, atraer personas mejor calificadas a la educación secundaria. Ello requiere incrementar los salarios generales de los profesores, acompañado de mayores responsabilidades profesionales y rendición de cuentas. Deben diseñarse incentivos alrededor del escalafón docente y otros, de manera que propicien una buena enseñanza.

El contenido de la preparación docente exige una completa reestructuración. Se requiere, por lo tanto, una política nacional dirigida a las instituciones de formación y capacitación 
docente, que se dirija a financiar innovaciones. La reforma de los programas de formación y capacitación debe incluir, entre otros, programas efectivos de enseñanza práctica; la incorporación apropiada de la tecnología en la enseñanza; y ofrecer incentivos a los mejores profesores universitarios en ciertas áreas para que se involucren en la capacitación y formación docente en servicio.

3. Reforma de la administración y del sistema escolar: Gran parte de la investigación reciente sobre escuelas eficientes hace énfasis en el papel fundamental del director de la escuela y en la importancia de la autonomía local, junto con la obligación de rendir cuentas a nivel de todo el sistema y la creación de incentivos para un mayor aprendizaje y/o retención de estudiantes. Sin embargo, poca investigación de este tipo se lleva a cabo en la Región. Entre los problemas críticos de la educación pública secundaria se señala su abandono por gran parte de la clase media y por la totalidad de la clase alta. Se propone la creación de escuelas "imán” o dedicar una parte de la escuela específicamente a ciertas áreas como matemáticas y ciencias, música y arte, o humanidades, que impartan un nivel de enseñanza equivalente al de las mejores escuelas privadas, pero con un costo sólo ligeramente superior al de las escuelas públicas, que ofrezcan especial atención a niños con talento provenientes de las familias más pobres.

4. Uso apropiado de la tecnología y de materiales de aprendizaje para el mejoramiento de la calidad: Se propone utilizar el potencial de la tecnología para incrementar el acceso y mejorar la enseñanza, así como exponer a los estudiantes a su utilización apropiada como parte de su educación general.
5. Reformulación de la estructura de la educación secundaria y de su relación con el mundo laboral: Plantea la necesidad de reducir la brecha entre los currículos vocacionales y los académicos. La educación secundaria en la Región ya no puede darse el lujo de desconocer la importancia cada vez mayor de competencias y habilidades diferentes de las académicas. Los objetivos de aprendizaje deberán incluir una mayor capacidad para la formulación y resolución conjunta de problemas; para el trabajo en equipo; responsabilidad cívica en una sociedad democrática; creatividad e innovación; así como una comprensión del papel de la tecnología en la sociedad, en especial de las tecnologías de la información, así como un conocimiento práctico de las computadoras. Se conoce poco acerca de cómo alcanzar estos objetivos, por lo que se torna necesario desarrollar aproximaciones menos costosas para alcanzarlos.

6. Realización de investigación aplicada sobre aquellos aspectos que funcionan bien en términos de aprendizaje, retención y desempeño en el mercado laboral: Destaca la importancia de sustentar las políticas educativas y la implementación de nuevas ideas y programas en los sistemas educativos, con base en investigaciones sobre los aspectos que funcionan bien. A partir de una revisión de investigaciones recientes, adelantadas en la Región, propone una lista de investigaciones que se requieren en educación secundaria, la cual incluye una variedad de temas y perspectivas. Entre ellas, menciona las siguientes:

6.1 Medición de los aspectos que funcionan mediante análisis estadísticos

6.2 Estudios sobre la eficiencia de las instituciones para determinar las variables críticas 
del proceso que inciden en un mejor rendimiento y retención de estudiantes

6.3 Estudios sobre demanda de profesores, conocimiento $\mathrm{y}$ pedagogía

6.4 Análisis del aprendizaje y del rendimiento en la escuela, así como del desempeño laboral de quienes abandonan los estudios, a efectos de determinar la pertinencia de la educación secundaria para el desempeño laboral

6.5 Análisis de los factores que inciden en la administración y gestión de los centros escolares.

\section{IV. ¿Desde dónde realizar la lectura e interpretación de las demandas y retos que se plantean a la educación secundaria?}

Ante el amplio panorama de retos y dilemas que enfrenta la educación secundaria en los sistemas educativos del mundo actual, tanto en los países más prósperos como en los más pobres, se torna indispensable realizar su lectura e interpretación estableciendo claramente las perspectivas que la informan. Tales perspectivas deben necesariamente constituir un referente conceptual que integre los aportes de aquellos estudios que, desde distintas miradas y enfoques, han abordado los problemas más relevantes a los que se pretende responder, y posicionarse frente a los argumentos que respaldan perspectivas distintas o contradictorias.

La construcción de dicho referente es fundamental para integrar una perspectiva profunda, que supere la visión superficial de retos y alternativas a la que se reduciría la lectura de este panorama de retos y dilemas, si se hace exclusivamente desde las creencias y perspectivas determinadas por la experiencia profesional en un determinado ámbito, independientemente de cómo se halle éste vinculado con la educación secundaria.

Seguidamente, se plantean aquellos conceptos que se consideran prioritarios para la construcción de dicho referente, los cuales, a su vez, sustentan los lineamientos que posteriormente se proponen para orientar los esfuerzos dirigidos a atender las diversas demandas y retos a los que actualmente se enfrenta la educación secundaria costarricense:

\section{El carácter histórico, complejo e interdependiente del sistema educativo}

El sistema educativo, en cualquier país, es un producto de su historia. Sus rasgos y peculiaridades tienen un origen concreto. Se estructura como una realidad institucional y pedagógica compleja, compuesta de prácticas un tanto autónomas, pero interdependientes, que mantiene unas relaciones con el sistema social externo. Por lo tanto, para comprender la situación que atraviesa la educación secundaria y estar en condiciones de proponer alternativas para el futuro, es necesario advertir las similitudes y diferencias entre los procesos educativos y su contexto social (Gimeno, 1997). De ahí que un análisis significativo y realista de las alternativas de cambio educativo requiere algo más que el simple balance de ventajas e inconvenientes de reformas concretas, ya que precisa examinar la relación de la parte con la totalidad -una reforma determinada con el fin que persigue y el contexto en que se desarrolla-.

\section{Las implicaciones de las condiciones sociales en que se inserta la educación secundaria y las propias de la institución escolar}

El contexto latinoamericano en el que se inserta nuestro país se caracteriza por el 
crecimiento económico con exclusión social, particularmente de los jóvenes. Cuando el sistema escolar se encuentra inserto en estrategias de desarrollo social excluyente, contribuye a fortalecer la exclusión juvenil. Si el sistema escolar es expulsor, se convierte en parte del riesgo estructural en que se desarrolla la población. Por lo tanto, en el análisis de los retos que enfrenta la educación secundaria es fundamental considerar la necesidad de que ésta contribuya a la construcción de la equidad (Puiggrós, 94; Krauskopf, 2001).

Por otra parte, si bien la cultura escolar enfatiza lo intelectual sobre la dimensión social, afectiva, estética, motriz o ética de los estudiantes, las instituciones escolares constituyen contextos integrales de desarrollo humano, por lo que su tarea incluye las dimensiones afectiva y social. Al insertarse en una institución escolar, ésta impone ciertas lógicas de funcionamiento que el joven debe resignificar seleccionando aquellos elementos que encajan con su propio relato construido. La educación afectiva y social que ocurre en las escuelas y liceos está implícita en cada institución y práctica escolar "formando una manera de sentir y de tratar a los seres humanos y al conocimiento" (Mena, et. al., 1993). Por lo tanto, si la expectativa se reduce a transmitir el conocimiento al margen de lo afectivo y lo social, el resultado será que los jóvenes no lo interioricen, provocando que funcionen disociadamente.

\section{La superación del paradigma de la universalidad de los programas mediante respuestas fundamentadas en nuevos paradigmas de desarrollo}

Desde la perspectiva de la equidad, el progreso en la cobertura de la educación debe acompañarse por la modificación del paradigma de la universalidad de los programas. La universalidad, entendida como homogeneidad programática, no logra impactar la marginación de los grupos menos favorecidos -etnias, rurales, urbano populares- porque no es una vía suficientemente diferenciada para lograr los niveles de aprovechamiento requerido. Por lo tanto, la homogeneidad de planteamientos educacionales que desconocen la diversidad, incrementan la discriminación de oportunidades, llevando a un círculo vicioso donde se excluyen los jóvenes en general, y en particular a los niños y adolescentes en pobreza, a las mujeres jóvenes, a las indígenas y a la juventud rural.

En consecuencia, la cultura homogénea del currículo, organizada alrededor de una estructura organizativa que gradúa lineal y rígidamente el itinerario escolar de quienes se benefician de las acreditaciones que otorga la escolaridad; la escasa variedad de espacios, de estímulos, y de recursos culturales para el aprendizaje, que favorecen el uso de fuentes uniformadas de información; junto a la carencia de respuestas fundamentadas en nuevos paradigmas de desarrollo, limita las posibilidades de admitir intereses, estilos, ritmos de aprendizaje, y formas de trabajar distintas dentro de un mismo grupo de alumnos, lo cual puede impactar riesgosamente las opciones y aspiraciones de jóvenes y adultos.

Los desafíos que enfrenta el sistema educativo demandan una respuesta a la rápida transformación cultural derivada de los cambios político-económicos mundiales y locales. En ese contexto, la multiculturalidad se asume con distintos ritmos por las diversas generaciones, en los distintos estratos socioeconómicos y culturas. Esto se agudiza en una época en que el futuro, como única meta ordenadora de los aprendizajes y sentido de vida en la etapa juvenil, ha perdido preeminencia (Krauskopf, 2001). De ahí que el modelo curricular común debe incorporar la perspectiva multicultural de manera que la integración de culturas se realice dentro de un sistema de escolarización única, que favorezca la igualdad de oportunidades. De lo contrario, sólo se posibilita la asimilación de unos por otros. 


\section{El carácter institucional del sistema educativo demanda que los cambios deseados se construyan como producto de procesos que incidan en la "cultura de lo pedagógico", y dispongan tanto de los medios para alcanzarlos como de una adecuada reorganización escolar}

La velocidad de los cambios afecta de diverso modo la temporalidad en la vida social; no es única, ni uniforme. El sistema educativo es una de las instituciones más difíciles de modificar, pues se enfrenta a la velocidad de la reestructuración económica y cultural desde la estructura de su institucionalidad. Es el producto y se transforma evolutivamente a través de modos de gestión cotidiana en un proceso en el que, por la vía de los hechos, condensa usos y tradiciones en una determinada "cultura de lo pedagógico", la cual pasa a formar parte de la realidad social y de los hábitos de una determinada sociedad (Parra, 1998; Gimeno, 1997).

Desde esa perspectiva, el sistema educativo y su dinámica no están determinados exclusivamente por las políticas y leyes educativas, ni tampoco por la ciencia y el conocimiento especializado, sino que se fraguan paulatina y parsimoniosamente, por lo que las iniciativas y declaraciones que, en forma ritual, cada determinado tiempo aparecen, logran en realidad muy pocas transformaciones. Los intentos de reforma generalmente se dirigen a lograr una mejor calidad. Sin embargo, lograrla requiere invertir medios para mejorar un sistema que, en su fase expansiva, logró únicamente cubrir las exigencias mínimas de materiales y de recursos humanos. De ahí que en momentos de crisis en el gasto social, el discurso sobre la calidad se restringe a unos significados más estrictamente eficientistas y a argumentos técnicos que no llegan fácilmente a los docentes; de esta forma, reemplaza a los medios reales para lograr un cambio: la "reconversión" de la competencia cultural, científica y pedagógica de los profesores, la dotación de medios didácticos alternativos, y la adecuada reorganización escolar.

Aquellas propuestas y programas de cambio que pretendan superar el nivel de discurso para lograr algún nivel de impacto en la realidad educativa, deberán reflejarse en la vida y funcionamiento de los establecimientos escolares. Es el centro escolar el que establece el ambiente general de aprendizaje para los alumnos e imprime su sello propio al desarrollo del currículo, y donde se genera la coordinación o el individualismo en las actuaciones profesionales; es ahí donde se concretan las condiciones de trabajo de los docentes, y donde estos deberán desempeñar nuevos roles y evidenciar nuevas competencias profesionales. Tal como señala Gimeno Sacristán (1997):

La práctica pedagógica se explica por las determinaciones y por el acomodo a una estructura organizativa; lo que no se cambie en este ámbito reduce el margen de innovación de los educadores al ambiente restringido de las aulas (p. 37).

\section{El desarrollo del profesorado como componente fundamental a considerar}

La importancia de la formación permanente del profesorado tiene una justificación en sí misma, en tanto que la enseñanza es una actividad social que, al igual que las demás, debe responder a cambios constantes que requieren respuestas apropiadas por parte de todos los elementos que inciden en la realidad educativa, siendo el docente uno de los más decisivos. De ahí que debe asumirse como una tarea permanente, la cual se torna más urgente en contextos de innovación o cambio. La atención profunda a los procesos de desarrollo del profesorado aparece como condición indispensable para que las innovaciones por promoverse no se queden en un nivel superficial y logren adentrarse en el núcleo 
de cómo aprenden los jóvenes y cómo enseñan los docentes.

Uno de los acuerdos que goza hoy de mayor aceptación es que el mejoramiento de la calidad de la educación pasa por docentes de calidad, debidamente seleccionados y motivados profesionalmente, con unas condiciones de trabajo que les permitan ejercer una enseñanza que se evalúa y se mejora constantemente, en una práctica compartida en equipos de trabajo, que cuente con los materiales y recursos oportunos (OCDE, 1991).

La participación de los profesores es vital para que el cambio educativo tenga éxito, sobre todo si éste es complejo, se desea generalizar, y es de largo plazo. Sin embargo, existen diversos modelos para aproximarse al desarrollo profesional que responden a distintas premisas respecto de su papel en la definición y conceptuación de las prácticas educativas y de sus deseos de perfeccionamiento y cambio, los cuales definen los instrumentos políticos y administrativos utilizados para realizarlo.

Hargreaves (1996) analiza algunos esfuerzos realizados recientemente, sobretodo en los Estados Unidos, dirigidos a reformar a los profesores y la enseñanza, a partir de discutibles hallazgos de investigación educativa, en los que se resta valor a la visión práctica y a la experiencia de los profesores, exigiéndoles que acepten el saber, la pericia y las normas que son propiedad y prerrogativa de científicos "expertos". Al respecto, menciona que como consecuencia de ello, los cambios propuestos han topado con el escepticismo y las quejas de los docentes sobre lo que consideran cambios caprichosos que responden a modas y novedades educativas, oscilantes, y por consiguiente, existe el peligro de que algunos de ellos acaben con cuestiones verdaderamente importantes, aunque no sean novedosas. Por el contrario, señala que la rica reserva bibliográfica de investigación y de comprensión práctica del proceso de cambio, evidencia que los esfuerzos de desarrollo del profesorado que muestran mayores indicios de reconocimiento y posibilidades de éxito, son aquellos que poseen las siguientes características: se realizan en el ámbito del centro escolar; consideran diversas opciones; son respetuosos del criterio de los profesores, en lugar de imponer fórmulas estandarizadas; y se desarrollan en un contexto de compromiso con el aprendizaje y el perfeccionamiento continuos.

Por lo tanto, la comprensión de los deseos de cambio y de conservación o resistencia de los profesores frente a éste, así como de las condiciones que fortalecen o debilitan esos deseos, ofrece una perspectiva importante de tener en cuenta. Ello no significa apoyar y celebrar todo lo que piensen, digan o hagan, pero sí tomar en serio sus percepciones y perspectivas.

Lamentablemente, los modelos de desarrollo del profesorado que han predominado, responden a una perspectiva carencial, que procura suplir las carencias de los docentes para responder a las nuevas demandas planteadas desde afuera, y así intentar modificar su actuación, con miras a la mejora de la calidad del proceso. Dichos modelos se sustentan en una perspectiva que ubica al profesorado como el principal referente que incide en la calidad de los resultados, dejando por fuera la consideración de todo el conjunto de prácticas concurrentes y simultáneas que inciden en ella, además de las condiciones de implantación de las propuestas de cambio.

\section{El tipo de investigaciones que deben sustentar las innovaciones y cambios propuestos}

Es necesario valorar las perspectivas en que se sustentan las investigaciones cuyos resultados se utilizan para respaldar innovaciones y cambios, de manera que no constituyan simplificaciones de la realidad educativa, pues de ser así, sus posibilidades para generar resultados que respalden propuestas no superficiales o pasajeras, son muy limitadas. 
A continuación se presenta una síntesis de los argumentos ofrecidos por Reynolds y Parker (1992) para señalar las limitaciones de la investigación y la práctica de las escuelas eficaces, con el propósito de destacar algunos aspectos importantes de tener en consideración en dicha valoración:

- Las listas de verificación de características no dan una idea clara y holística de lo que hace eficaz una escuela ni de cómo se establece. También es preciso conocer a qué pautas responden estas listas y cómo se integran en forma de una cultura global de la escuela.

- Aunque sepamos a qué se parecen las escuelas eficaces, no sabemos cómo crearlas. Algunas tentativas para generar escuelas de este tipo sobre la base de los resultados de investigación han generado a menudo la oposición de los profesores ante lo que perciben como un cambio impuesto, no deseado y que supone un exceso de trabajo.

- Hay cantidad de evidencia que da cuenta de que las escuelas eficaces no permanecen necesariamente estables en el tiempo, por lo que aunque supiésemos cómo crearlas, todavía debemos determinar cómo mantenerlas.

- Las escuelas eficaces suponen una definición restringida y convencional de la eficacia, que subrayan los resultados académicos y destrezas básicas, pero no necesariamente responden a las necesidades del entorno en términos de la creatividad, resolución de problemas, el manejo del riesgo, aprender a aprender y la flexibilidad.

- La eficacia responde con frecuencia a características contextuales y culturales específicas limitándolas, por lo tanto, a ambientes similares a los estudiados.

\section{La "flexibilidad" como una oportunidad democrática abierta}

Con respecto a los tipos de organización, la sabiduría colectiva de la bibliografía contemporánea, destaca que los tipos de organización con más probabilidad de prosperar en el mundo actual, son aquellas caracterizadas por la flexibilidad, la adaptabilidad, la creatividad, el aprovechamiento de las oportunidades, la colaboración, el perfeccionamiento continuo, una orientación positiva hacia la resolución de problemas y el compromiso para maximizar la capacidad de aprender sobre su ambiente y sobre ellas mismas.

Por el contrario, la mayoría de las estructuras escolares existentes, sobre todo en el nivel de educación secundaria, se caracteriza por formatos de clase única, aula única, profesor único, por lo que no están preparadas para responder a las necesidades organizacionales que hoy se requieren.

Sin embargo, es necesario señalar la necesidad de procurar el objetivo de la flexibilidad, desde una perspectiva crítica orientada hacia la equidad, que evite generar beneficios para algunos sectores sociales y sus intereses, por sobre los otros. Ello requiere atender a sus diversos significados para los distintos sexos, clases sociales y etnias presentes en el conjunto de la fuerza de trabajo; analizar sus consecuencias sobre el desempleo, el subempleo y el empleo de bajo nivel, al que necesariamente deben acceder muchos trabajadores en función de las categorías o estratos en que se les ubica, en razón de sus diferencias con respecto a aquellos considerados superiores. Esto, por cuanto la "flexibilidad" puede crear ambientes de trabajo y estructuras sociales elitistas, reservando la autonomía, el criterio propio y el trabajo más significativo para las pequeñas elites técnicas, por lo que el trabajo flexible y enriquecedor es calificado de manera diferente por los trabajadores. 
En este sentido, para los profesores y demás personas que trabajan con ellos, es importante considerar la "flexibilidad" como una oportunidad democrática abierta que exige compromiso y participación crítica y no una obligación institucional cerrada que demanda aceptación completa.

\section{El desarrollo de un sistema de evaluación que responda a una concepción amplia del fracaso escolar}

Finalmente, es conveniente analizar los esfuerzos por mejorar los aprendizajes y evitar el fracaso escolar acompañados de medidas del rendimiento, desde una concepción que reconoce el fracaso escolar como un fenómeno históricamente reciente, ligado a la aparición en nuestras sociedades de una institución encargada de impartir ciertos aprendizajes a un grupo determinado de edad, según unas modalidades codificadas -normas relativas a lugares, calendario, contenido y segmentación, edades de los estudiantes, organización de la clase, sistema de evaluación y certificación, entre otros-. Ello permite tomar conciencia de que el fracaso no se limita a las dificultades de aprendizaje del estudiante, sino además, de alguna manera, conlleva el reconocimiento oficial de estas dificultades: es lo que dice la escuela acerca del alumno o lo que hace la escuela con el alumno con problemas.

De ahí que, si bien el fracaso se ubica en individuos particulares, a menudo los factores que lo provocan quedan fuera de control y responsabilidad. ¿Quién fracasa en realidad? Fracasan los individuos, fracasa la sociedad, la escuela y las políticas educativas. Por lo tanto, es importante estudiar el fenómeno del fracaso y analizar las propuestas de evaluación de los aprendizajes desde perspectivas que no simplifiquen y reduzcan la problemática. Esto facilitará el reconocimiento de la necesidad de dedicar esfuerzos a mejorar los sistemas de evaluación y rendición de cuentas.

\section{A manera de conclusión: algunos lineamientos para orientar los esfuerzos dirigidos a atender las diversas demandas y retos a las que actualmente se enfrenta la educación secundaria costarricense}

Del panorama aquí trazado, se desprende que el mayor desafío que enfrenta la educación secundaria en Costa Rica es recoger y analizar los diversos aportes generados a partir de las distintas reflexiones, experiencias y esfuerzos realizados en torno a ella, con miras a replantearla de una manera profunda e integral, que responda a unas perspectivas claramente delineadas, que superen las interpretaciones superficiales y simplistas en relación con los cambios y transformaciones que hoy se demandan de ella.

Desde el referente conceptual aquí propuesto, es posible identificar un conjunto de líneas de acción que deben atenderse con prioridad, a partir del reconocimiento de las siguientes apreciaciones acerca de la educación secundaria costarricense:

- $\quad$ Es producto de una realidad histórico-cultural y socialmente situada, que se integra en un sistema educativo complejo e interdependiente, tanto en su interior como con el contexto en el que se inserta. Por lo tanto, es indispensable atender tanto su dinámica histórico-social como sus relaciones con el sistema en su totalidad.

- Las características de inequidad y exclusión social en que se desarrolla, tornan indispensable la recuperación del espacio educativo formal de la escuela pública, del cual son excluidas grandes cantidades de jóvenes, especialmente aquellos provenientes de los grupos más desfavorecidos. Para ello, es indispensable propiciar la construcción de enfoques que 
incorporen las diferentes realidades socioeconómicas y culturales en que se encuentran insertos los estudiantes, como punto de partida para desarrollar estrategias educativas plurales y complementarias, superando la división de educación formal e informal.

- Debe garantizar a los jóvenes oportunidades para el diálogo y la colaboración intergeneracional, así como para involucrarse en servicios de la comunidad, propiciando interacciones cara-a cara que faciliten la construcción de puntos de vista propios, para apoyar así el desarrollo del proceso de construcción de identidad, en el que se encuentra la población que accede a ella. Esto requiere una adecuada gestión y coordinación de esfuerzos de la familia, los docentes y de las facilidades que ofrezca el entorno.

Como conclusión, se exponen a continuación las líneas de acción que se proponen como prioritarias para atender los principales retos que enfrenta hoy la educación secundaria en nuestro país:

1. La reformulación conceptual del modelo vigente, como parte de un proceso continuo, a efectos de replantear sus objetivos y propósitos, de manera que ésta resulte pertinente para las necesidades y expectativas del entorno en que se inserta. Las expectativas a las que debe responder la educación secundaria costarricense son muy distintas a aquellas en las que se gestó el modelo existente. Si bien es fácil lograr consenso en torno a declaraciones genéricas en torno al modelo educativo al que debe responder, no lo es el establecimiento de formulaciones concretas. De ahí que es necesario construir un modelo operativo que permita identificar los requerimientos concretos derivados de una experiencia educativa en la que se espera la educación del ciudadano, la búsqueda del espíritu creativo y crítico en los alumnos, la educación ética y afectiva, la atención a la diversidad en un contexto de inequidad, así como una flexibilidad tal que permita trazar diversos caminos para alcanzar una metas educativas comunes, entre otros. Dicha reformulación debe ser capaz de lograr ponderar, equilibrar y balancear expectativas contradictorias que responden a perspectivas en debate, en torno a la búsqueda de la equidad y del papel que la educación secundaria pública tiene en una sociedad como la nuestra.

2. Diseñar e impulsar la construcción de procesos que se sostengan en el mediano y largo plazo, orientados a promover la reorganización de las instituciones en las que ésta se concreta, así como al desarrollo de alternativas curriculares viables, que respondan a las expectativas y necesidades identificadas en los esfuerzos de reformulación de su modelo. Ello, para procurar una mejor articulación con las demás instituciones de su entorno, en la búsqueda de integrar verdaderas comunidades de aprendizaje por un lado, y por otro, para buscar la flexibilidad curricular que se requiere para atender la diversidad de la población que accede a ella. Además, estos procesos deben dirigirse a lograr un balance apropiado entre la formación de habilidades y destrezas de nivel superior y la de actitudes y competencias para la inserción laboral, en el marco de la búsqueda de equidad y del reconocimiento de la importancia de la participación democrática de todos los involucrados, de manera que no resulte una imposición desde afuera.

3. Favorecer el desarrollo profesional de los profesores mediante su participación en proyectos de desarrollo profesional que se sustenten en prácticas 
desarrolladas en el centro escolar, para los que cuenten con el tiempo, incentivos y materiales, así como con el acompañamiento necesario de otras instituciones y actores, por medio de grupos colaborativos multidisciplinarios de trabajo, no impuestos. Esto requiere superar el modelo de "profesor taxi" que predomina en nuestros colegios y liceos, y priorizar y valorar la formación docente inicial y en servicio.

4. La necesidad de realizar investigaciones sistemáticas desde perspectivas que resulten sensibles a las culturas institucionales, que consideren la complejidad, el carácter histórico y la interdependencia del sistema educativo tanto a lo interno como con el entramado social en que se inserta. Ello, con el propósito de generar estrategias e innovaciones didácticas que respondan a las características de los contextos en que se inscribe la educación secundaria, así como nuevas y más apropiadas formas de evaluación, que valoren de manera integral los distintos determinantes del rendimiento, y que puedan ser utilizadas para mejorar las prácticas, en vez de simplemente identificar el fracaso de estudiantes, profesores e instituciones.

5. El desarrollo de las líneas de acción mencionadas, requiere de una importante inversión de recursos humanos, financieros y materiales, lo que a su vez genera la necesidad de prever y gestionar un conjunto de esfuerzos y acciones indispensables para hacerla efectiva, en un entorno que reclama la reducción del gasto público y de la intervención estatal.

\section{Referencias bibliográficas}

Contreras, Ileana. (2002). "La educación matemática en la escuela secundaria: Mecanismos de resistencia a un modelo agotado". Informe de investigación Proyecto No. 724-97-347, IIMEC, Universidad de Costa Rica.

Donas, Solum. (2001). "Adolescencia y juventud: Viejos y nuevos desafíos en los albores del nuevo milenio". En Adolescencia y Juventud en América Latina, Solum Donas Burak (Compilador). Cartago, Libro Universitario Regional.

Elmore, Richard. (2003). "La reforma escolar y la nueva economía”. En El fracaso escolar: Una perspectiva internacional, Álvaro Marchesi y Carlos Hernández (Coordinadores). Madrid: Alianza Editorial, S.A.

Gimeno, José. (1997). Docencia y cultura escolar: Reformas y modelo educativo. Buenos Aires: Lugar Editorial.

Hargraeves, Andy. (1996). Profesorado, cultura y postmodernidad. Madrid: Morata.

Krauskopf, DINA. (2001). "Los nuevos desafíos de la educación en el desarrollo juvenil". En Adolescencia y Juventud en América Latina, Solum Donas Burak (Compilador). Cartago, Libro Universitario Regional.

Leclercq, Jean-Michel. (2001). "La educación secundaria general en el siglo XXI: Desafíos, tendencias y prioridades". Documento de trabajo para la Reunión de Expertos Internacionales sobre educación secundaria en el siglo XXI. Informe Final, Beijing: UNESCO.

Mead, Margaret. (1971). Cultura y compromiso. Granica: Buenos Aires.

Mena, M., Arancibia, V.Y. Bruzzone, M. (1993). Desarrollo socioafectivo en la enseñanza media. Santiago: CPU. 
Obiols, Guillermo y Obiols, Silvia. (1995). Adolescencia, posmodernidad y escuela secundaria: La crisis de la enseñanza media. Buenos Aires: Kapelusz Editora.

OCDE. (1991). Escuelas y calidad de la enseñanza. Barcelona: Paidós-MEC.

Parra, Rodrigo. (1998). "El tiempo mestizo. Escuela y modernidad en Colombia" citado por Dina Krauskopf en Adolescencia y Juventud en América Lati$n a$, Solum Donas Burak (Compilador). Cartago, Libro Universitario Regional.

Puiggrós, Adriana. (1994). "América Latina y la crisis de la educación". En Alternativas pedagógicas, Adriana Puiggrós y Marcela Gómez (Coord). Buenos Aires: Niño y Dávila.

Reich, Robert. (1992). The work of nations. Nueva York: Random House, p. 3.

Reynolds, David y Parker, A. (1992). "School effectiveness and school improvement in the 1990s" en School effectiveness: Research, policy and practice, D. Reynolds y P. Cuttance (Editores). London: Cassell.

Roldán, Cándido. (2001). "Desarrollo de adolescentes y jóvenes en zonas de pobreza y marginación". En Adolescencia y Juventud en América Lati$n a$, Solum Donas Burak (Compilador). Cartago, Libro Universitario Regional.

CONARE. (2005). "Universalización de la educación secundaria y reforma educativa". En Undécimo Informe sobre el Estado de la Nación en Desarrollo Humano Sostenible. Recuperado de www.conare.ac.cr.

Wolff, Laurence y de Moura, Claudio. (2000). Educación Secundaria en América Latina y el Caribe: los retos del crecimiento y la reforma. Washington DC: Banco Interamericano de Desarrollo ( No. EDU. 111). 
